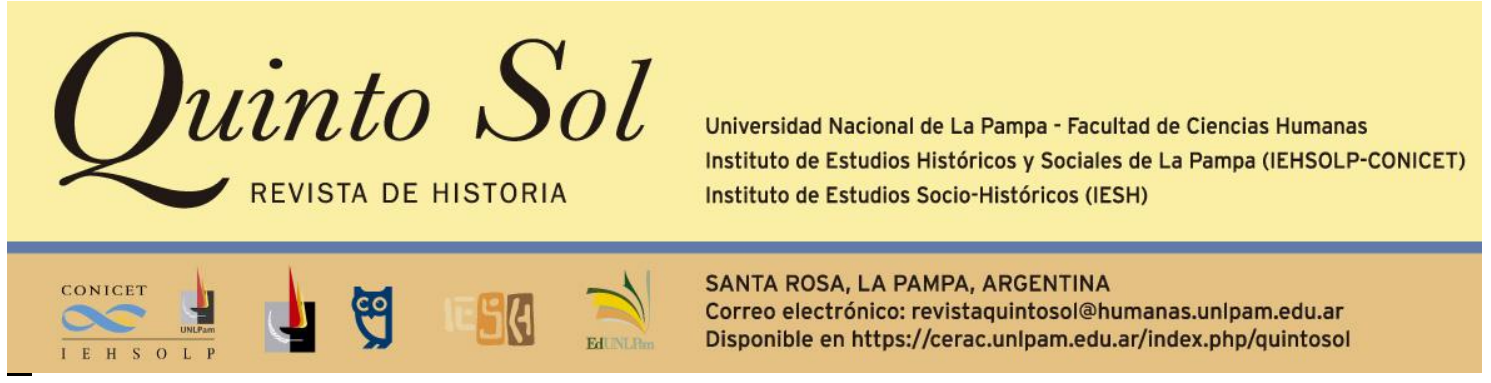

Quinto Sol, vol. 25, n², mayo-agosto, ISSN 1851-2879, pp. 1-9

http://dx.doi.org/10.19137/qs.v25i2.5801

Esta obra se publica bajo licencia Creative Commons 4.0 Internacional. (Atribución-No Comercial-

Compartir Igual)

\title{
"El Estado a veces hace políticas que se contradicen entre sí". Las políticas públicas cooperativas (2008- 2015). Entrevista a Mario Lattuada
}

\author{
Rocío Soledad Poggetti \\ Consejo Nacional de Investigaciones Científicas y Técnicas \\ Universidad Nacional de Río Cuarto. Instituto de Investigaciones Sociales, Territoriales y Educativas \\ Universidad Nacional de Córdoba. Centro de Investigaciones "María Saleme de Burnichon" \\ Argentina \\ Correo electrónico: rpoggetti@hum.unrc.edu.ar
}

\section{Introducción}

Los estudios cooperativos se han ocupado de indagar acerca de una diversidad de dimensiones que forman parte de la dinámica de las cooperativas agropecuarias. Entre los más relevantes podemos mencionar el origen histórico de estas asociaciones en relación con la expansión de la frontera agropecuaria, la vinculación entre la estructura organizativa e institucional y los principios cooperativos, las tensiones o las contradicciones entre la doctrina y las prácticas empresariales, la expansión de estas asociaciones en el territorio nacional, los programas de educación cooperativa, las transformaciones en las bases sociales en relación con las mutaciones del modelo agrario durante el siglo XX, las políticas públicas que han incidido en el desarrollo del cooperativismo, entre otros (Lattuada, 2002; Girbal de Blacha, 2002, 2003; Mateo, 2012; Olivera, 2015). 
En relación con esta última, los académicos/as se concentraron en la descripción de las políticas que incidieron (y que lo hacen en la actualidad) en el cooperativismo agropecuario, con particular énfasis en la coyuntura del peronismo histórico (Lattuada, 2002; Girbal de Blacha, 2002; Olivera, 2015). Los esfuerzos iniciales en este sentido han procurado discernir los niveles político-institucionales en los que se inscriben, sea nacional, provincial, regional y local, o el sector sobre el cual intervienen -economía, finanzas, agro, cooperativas- (Lattuada, 2006; Olivera, 2015). A partir de mediados de la primera década del siglo XXI, en el marco de la complejización de los estudios referidos al Estado (Bohoslavsky y Soprano, 2010), es notorio el esfuerzo de las y los investigadores por incorporar la institucionalidad en la que se inscriben los procesos de gestión e instrumentación de las políticas públicas (Nogueira, Urcola y Lattuada, 2017). En algunos de estos casos, se introducen cuestiones vinculadas a la jerarquía de las agencias abocadas a la gestión de las políticas cooperativas, su inserción institucional, los referentes políticos que se encuentran a cargo de las mismas, la asignación presupuestaria, entre otras cuestiones.

Nos interesa destacar dos aspectos que se evidencian en estos análisis. Por una parte, es posible advertir un reconocimiento explícito en algunos casos e implícito en otros, acerca del condicionamiento que ejercen las políticas públicas en la dinámica del movimiento cooperativo agropecuario en general y en las estructuras organizativas e institucionales de estas asociaciones en particular. Por otra parte, existe una tendencia a analizar las tramas estatales como espacios porosos, conflictivos e inestables. En este sentido, se procura indagar en las tensiones o contradicciones entre las agencias y las políticas públicas que directa e indirectamente inciden sobre las cooperativas.

En este marco, nos interesa compartir la entrevista que realizamos al Dr. Mario Lattuada, en tanto nos permite reflexionar acerca de estas cuestiones durante la coyuntura que el autor denomina como "régimen social de acumulación de economía regulada" (Lattuada et al., 2011). En este sentido, advertimos que tiene una doble potencialidad. Procura dar los pasos iniciales en la descripción de las políticas cooperativas durante la gestión kirchnerista, en particular durante el período 20082015, y además reconoce que esas políticas fueron diversas. Dentro de esta heterogeneidad identifica dos universos. Uno, vinculado a las políticas de flexibilización de la normativa cooperativa y la asignación de recursos para las asociaciones que nucleaban a agricultores familiares, tales como la reducción del número mínimo de asociados, eximición de presentar cierta documentación contable, entre otras (Lattuada, $2014^{\mathrm{a}}$ y $2014^{\mathrm{b}}$ ). Otro, relacionado a aquellas políticas y medidas más dispersas que tenían como objetivo aggiornar la normativa para que las cooperativas agroexportadoras y agroindustriales pudieran insertarse competitivamente en el agronegocio -algunas fueron la reglamentación de la vinculación con sociedades de capital, con terceros no asociados, integraciones, la formación de capital complementario-, muchas de las cuales habían sido introducidas por la Ley de Cooperativas 20.337.

La trama estatal de la que emergieron las políticas de fomento y acompañamiento de las cooperativas más vulnerables, así como las modificaciones en la normativa para favorecer el desarrollo de aquellas vinculadas a la agroexportación, puede ser caracterizada como compleja y cambiante. Esta complejidad debe ser inscripta en el marco de un régimen social de acumulación que procuró dinamizar la organización social y política de los sectores sociales -productivos en este caso- más 
vulnerables, pero que al mismo tiempo benefició a los sectores concentrados del agro al contribuir a la consolidación del agronegocio (Nogueira, Urcola y Lattuada, 2017). La conflictividad propia de esta coyuntura -y una de cuyas expresiones fue el conflicto entre el gobierno y los sectores agroexportadores así como las reminiscencias políticas y legislativas que evidenció la disputa entre los dos bloques pre-hegemónicos (Pucciarelli y Castellani 2014)- aporta un elemento sustancial para dilucidar las condiciones políticas e institucionales en las que se gestaron políticas cooperativas aparentemente contradictorias.

Para conocer aspectos de esta temática transcribimos la entrevista realizada a Mario Lattuada, especialista de vasta trayectoria en investigación, docencia y asesoramiento en el ámbito de las políticas públicas, las organizaciones profesionales y económicas empresariales, la economía social, los movimientos sociales y el desarrollo rural.

Lattuada es licenciado en Antropología Social y doctor en Humanidades y Artes por la Universidad Nacional de Rosario. Se desempeña como Investigador Principal del Consejo Nacional de Investigaciones Científicas y Técnicas (CONICET) y director del Centro de Altos Estudios en Ciencia Sociales de la Universidad Abierta Interamericana. Integra las Comisiones Asesoras en Ciencia y Tecnología del Consejo de Rectores de Universidades Privadas y de la Organización de Estados Iberoamericanos. Formó parte del directorio del CONICET y fue vicepresidente de Asuntos Tecnológicos (2001-2008). Entre 2008 y 2010 fue Subsecretario de Coordinación Institucional del Ministerio de Ciencia, Tecnología e Innovación Productiva de la República Argentina. En temas de su especialidad ha asesorado a diversos organismos e instituciones nacionales e internacionales, tanto públicas como privadas, tales como el Programa de Naciones Unidas para el Desarrollo, la Organización Mundial para la Agricultura y la Alimentación, el Banco Interamericano de Desarrollo, el Instituto Interamericano de Desarrollo Agrícola, el Ministerio de Agricultura, Ganadería y Pesca de la Nación, el Instituto Nacional de Asociativismo y Economía Social, la Federación Agraria Argentina, entre otras entidades. Es docente y se desempeñó como tal en universidades públicas y privadas nacionales y latinoamericanas, dirigió numerosas tesis de grado y posgrado, integró comités editoriales, de evaluación de instituciones y recursos humanos en ciencia y tecnología, consejos directivos, consultivos y académicos de diversas instituciones. El resultado de sus investigaciones se materializa en la publicación de 19 libros, 20 capítulos en libros y más de 60 artículos en prestigiosas revistas nacionales e internacionales. Actualmente dirige el proyecto de investigación titulado "La política agraria en los tiempos de la grieta. Argentina 2003- 2019" que aborda un estudio comparativo de los tres gobiernos que abarca ese período, los resultados serán publicados en un libro que se está elaborando y se editaría en el corriente año. 


\section{Entrevista}

\section{Pregunta: ¿Qué factores lo incentivaron a investigar el cooperativismo agropecuario desde la perspectiva de la acción colectiva?}

De alguna manera fueron confluyendo cuestiones académicas, los intereses de mis alumnos, otras más vinculadas a los estudios de campo y mi relación con Juan Mauricio Renold. Él es profesor e investigador en la Universidad Nacional de Rosario y se especializa en el análisis institucional de diferentes organizaciones, entre ellas cooperativas. Juntos compartimos, desde 1984, la cátedra de Introducción a la Antropología en la Facultad de Humanidades y Artes, en la misma casa de estudios.

En relación con lo primero, quiero destacar dos cuestiones. Por un lado, en 1994 comencé a coordinar la Maestría en Estudios Agrarios de FLACSO. Varios de los alumnos estaban vinculados a las cooperativas ya sea como productores, asesores 0 gerentes. Por el otro, en mi tardía tesis doctoral investigué las transformaciones de las organizaciones gremiales y empresariales del agro argentino durante el neoliberalismo. En particular, opté por tres tipos de organizaciones: la gremial cooperativa a partir de la Federación Agraria Argentina (FAA), los movimientos sociales -el caso de Mujeres Agropecuarias en Lucha- y el movimiento cooperativo. En este marco, comencé a orientar mis estudios desde los procesos hacia los actores. Es decir, pasé de estudiar las políticas agrarias en los partidos políticos a concentrarme en los actores de base para los cuales se gestionaban esas políticas y las estructuras de representación de los intereses en las cuales se insertaban.

En cuanto a lo segundo, durante varios años -1997-2001- coordiné un programa de desarrollo del Banco Interamericano de Desarrollo junto a FAA para las cooperativas de la región pampeana. En particular, se gestionaban recursos y proyectos para los pequeños productores de la región. Esto me abrió la posibilidad de hacer algunos trabajos de campo en cooperativas agropecuarias a las cuales estaban asociados esos productores.

En definitiva, se fueron superponiendo una serie de circunstancias que me incentivaron a estudiar las cooperativas agropecuarias tanto desde la perspectiva de la acción colectiva como desde un enfoque más estructural. Este tiene que ver con las mutaciones organizativas que se producen en las asociaciones no reivindicativas del agro $^{1}$ en el marco de las condiciones políticas, económicas y sociales propias de cada contexto.

\section{Vinculado a la propuesta conceptual que han construido con Juan Mauricio Renold para analizar la evolución histórica de las cooperativas agropecuarias ¿fueron las tensiones evidenciadas en el modelo organizativo de estas asociaciones las que animaron ese esfuerzo teórico?}

Parto por explicar, en líneas generales, las categorías que construimos. El objetivo era crear una tipología a partir de la propuesta weberiana de los tipos ideales que nos permitieran analizar las mutaciones organizacionales de las cooperativas. Surgieron de ese esfuerzo las siguientes: organización institucional consecuente (OIC), organización institucional paradojal (OIP) y las organizaciones institucionales en

\footnotetext{
${ }^{1}$ Las asociaciones no reivindicativas se constituyen con fines netamente económicos, su discurso no pretende interpelar a una población más amplia que su base social $y$, por lo general, no plantean reivindicaciones en el espacio público (Lattuada, 2006).
} 
mutación -de esta podía resultar una organización institucional de competencia dinámica (OICD) o un retorno a una organización institucional mutualista (OIM)-.2

En el contexto actual, es probable que predominen los últimos tipos de modelos organizativos. El cooperativismo que llamamos empresarial constituye una adaptación exitosa del modelo cooperativo a la lógica del agronegocio. El quiebre se produce cuando el interés institucional trasciende al interés de los asociados, que son en definitiva los propietarios y al servicio de quienes deben estar las cooperativas. La aparente tensión entre el bienestar de los asociados y el de la cooperativa en sí deja lugar a un proceso de complejización institucional que expulsa a aquellos eslabones más débiles de la cadena, que desde 1990 en adelante son los pequeños productores agropecuarios.

En estos casos, el control sobre el proceso de decisión queda reducido al staff general y un núcleo de la dirigencia que prioriza los componentes económicos, financieros y comerciales por sobre los sociales. Es decir, el gerenciamiento se transforma en un estamento en sí mismo que subsume la voluntad de los asociados a la lógica de la maximización económica. En consecuencia, la reducción de los espacios para que los socios productores intervengan en el proceso de toma de decisiones hace que disminuya su participación en las diversas instancias asociativas. La democracia queda desvirtuada debajo de los juegos de poder que demanda la reproducción institucional. No obstante, hubo un intento por parte de las políticas públicas cooperativas de fortalecer las experiencias asociativas y cooperativas de la agricultura familiar. En particular durante el período 2008-2015, en el marco del régimen social de acumulación de economía regulada.

\section{La política pública cooperativa se ha caracterizado históricamente por su inestabilidad ¿cuáles son las principales tendencias en política cooperativa que puede identificar desde 1989 hasta 2015?}

Creo que hubo distintas orientaciones en ese período. Es posible identificar al menos tres momentos claramente diferentes: 1989-2003, 2003-2008 y 2008-2015. El último es en particular interesante porque en cierta medida sintetiza los anteriores al tiempo que imprime un sello distintivo a la política pública. No solo porque se politiza la política cooperativa para el agro sino también porque se la incluye dentro de un proyecto más amplio como es la economía popular y social. El punto que da inicio a esta coyuntura es el conflicto con los sectores agroexportadores ${ }^{3}$ (2008) y finaliza con la administración de Mauricio Macri (2015), cuando la política cooperativa retoma

\footnotetext{
${ }^{2}$ Las OIC se encontraban asentadas principalmente en los valores cooperativos $y$, en consecuencia, se le otorgaba importancia a los factores sociales tales como la participación asamblearia de los productores, la cohesión, la solidaridad, la toma de decisiones democrática, el enraizamiento local, entre otras dimensiones. En cambio, la OICED desarrollaba diversos procesos de complejización institucional y diversificación territorial para adecuar la institución a los parámetros de un mercado agropecuario abierto y globalizado. Si en el tipo anterior predominan los aspectos micro del capital social, en este lo hacen los macro. En función a ello, disminuye la identidad, el vínculo asociativo se hace más pragmático, demuestran un menor grado de integración intracomunitaria y uno mayor con la comunidad local, nacional y global (Lattuada, et al., 2011).

3 Durante este conflicto, las entidades reivindicativas del agro -Sociedad Rural Argentina (SRA), FAA, Confederaciones Rurales Argentinas (CRA) y Confederación Intercooperativa Agropecuaria (CONINAGRO)(Lattuada, 2006) disputaban con el gobierno a raíz de la instrumentación de las retenciones y la transferencia intersectorial de ingresos (Pucciarelli y Castellani, 2014).
} 
mecanismos más tradicionales para gestionar y promover el funcionamiento de estas organizaciones.

Es posible reconocer durante el kirchnerismo dos principales tendencias en la política pública cooperativa -vinculada a la heterogeneidad del cooperativismo agropecuario-. Una, tiene que ver con la adecuación de la normativa cooperativa destinada a las cooperativas agroexportadoras y agroindustriales más grandes de la región pampeana. Otra, procura flexibilizar algunos preceptos legales para facilitar el proceso organizativo de los sectores vulnerables del agro.

Hay una tendencia que se hizo evidente en el desarrollo del cooperativismo agropecuario desde 1990. El crecimiento económico, comercial y financiero de estas asociaciones genera una serie de dificultades operativas que derivan de las limitaciones que imponen las normativas vigentes, en particular, las que emanan de la Ley de Cooperativas 20.337. Se ha tendido desde el Instituto Nacional de Asociativismo y Economía Social (INAES) a resolver sobre una serie de componentes con la finalidad de adecuar la legislación a las necesidades de estas cooperativas empresariales. También hubo medidas de apoyo a esas cooperativas más exitosas, tales como facilidades para los negocios en el exterior y líneas específicas de financiamiento.

Aunque también hubo un intento de facilitar la formación de cooperativas para una cantidad de sectores que no contaban con los recursos necesarios para organizarse, desde el punto de vista económico, legal y técnico. Desde este objetivo parten un conjunto de modificaciones que procuran flexibilizar las condiciones mínimas para la constitución y desempeño de estas. Entre ellas se pueden mencionar la reducción del número de asociados mínimos, de requisitos legales para la constitución, de documentación que deben presentar para certificar el desempeño, entre otras. Este proceso se acompañó de la implementación de algunos programas que procuraban la transferencia de recursos hacia estos sectores a través de la formación de experiencias asociativas que, si bien en un primer momento no eran cooperativas, tendían en el mediano y largo plazo hacia esta forma organizativa. Opté por denominar a estas heterogéneas formas asociativas pre-cooperativas como asociaciones económicas no cooperativas de la agricultura familiar, para contemplar la diversidad de formas que impulsó el Estado con el fin de contribuir al afianzamiento de estos sectores (Lattuada, $2014^{\mathrm{a}}$ y $2014^{\mathrm{b}}$ ).

Las políticas generadas por las diferentes agencias del Estado no siempre son coherentes entre sí. Así como pueden complementarse puede llegar a competir con objetivos diferentes y hasta contradictorios. Respecto de esta última tendencia es posible reconocer dos orientaciones en las políticas y los programas de acompañamiento para las asociaciones y las cooperativas de la agricultura familiar. Los programas de desarrollo rural llevados a cabo en este período promovían la integración de los integrantes de la agricultura familiar en diversas formas de asociaciones pre-cooperativas. Una que se orientaba más hacia la importancia de los componentes económicos de las organizaciones y otra que procuraba fortalecerlas en sus dimensiones sociales y políticas. En la primera tendencia es posible reconocer las medidas que dependían de la Unidad para el Cambio Rural, dependiente del Ministerio de Agricultura, Ganadería y Pesca; las segundas, en cambio, lo hacían de la Secretaría de Agricultura Familiar. 


\section{En relación con esta segunda orientación de la política cooperativa ¿es posible articularla con el proyecto político más amplio que representó el INAES?}

Debe considerarse que el sector agropecuario y el universo de las cooperativas -o del asociativismo en general- en Argentina son heterogéneos. Las políticas públicas para los sectores más vulnerables del agro recuperaron durante esta etapa la inserción en un proyecto institucional que se había diluido en $1990 .{ }^{4} \mathrm{Y}$ allí hay que ver las agencias que se abocaron específicamente a estas asociaciones. Pero también es necesario tener en cuenta el hecho de que las políticas que se gestionan para estos sectores se pueden articular con otras áreas institucionales del Estado. No solo el INAES gestiona las políticas para las cooperativas, sino que otras agencias del Estado también influyen en forma relevante sobre el devenir de las mismas. Creo que allí hay una potencialidad y una limitación de la política pública cooperativa propia de este contexto.

La política que se gestionó para el sector estaba impregnada por todo lo que conformaba la economía popular y las empresas recuperadas. Creo que no habría que quedarse con una mirada acotada solo al sector agropecuario, porque eso nos haría perder muchos de los sentidos que adquirieron las políticas para los agricultores familiares en este contexto; es decir, hay que incluirlas en una estrategia más general vinculada a la economía social. Cuando se produjo el cambio en la Secretaría de Agricultura Familiar, Ilamó la atención que designaran a alguien como Emilio Pérsico que provenía de los movimientos sociales, del Movimiento Evita, pero que no estaba vinculado al sector agropecuario. Entonces, lo que puede advertirse allí es una orientación más política y social que económica en los procesos de organización de los sectores más postergados del agro.

Sin embargo, era un modelo que en sí mismo estaba tensionado porque no necesariamente había una unidad de criterios dentro de las políticas cooperativas. Dependía de los grupos políticos y su orientación. Esto resta coherencia a la política cooperativa e impide construir acuerdos de mediano plazo que permitan gestionar no solo medidas de fomento sino también de acompañamiento técnico y financiero. En parte debido a esta contradicción, pero también vinculado a deficiencias de los mismos programas, muchas de las asociaciones o cooperativas que se crearon en este contexto no lograron sobrevivir al cambio de orientación política que sobrevino a partir de 2015. Entre los factores que hacen a esa discontinuidad puedo mencionar el ejemplo de algunas que no contaban con la estructura organizativa adecuada, otras que no tenían la dimensión de negocios necesaria o que se negaban a sumarse en proyectos que agregaban a más de una entidad. Aunque, en general ocurrió que los proyectos no eran sentidos como propios por quienes eran sus protagonistas.

\section{Entonces ¿considera usted que las políticas cooperativas deben adecuarse de acuerdo al tipo de organización cooperativa?}

Considero que no hay un modelo de políticas cooperativas al cual se pueda aspirar. Las condiciones de heterogeneidad ameritan la gestión de políticas focalizadas

\footnotetext{
4 Durante la década de 1990 los programas y las medidas de apoyo para los agricultores familiares dependía en lo fundamental de programas de desarrollo rural. Estos procuraban fomentar el asociacionismo con una lógica compensatoria, buscaban mejorar sus rentas a través de la diversificación productiva, el incremento en la escala y la participación en nuevos eslabones de la cadena alimentaria. Para ampliar el análisis de esta coyuntura, invitamos al lector a consultar el trabajo de Nogueira, Urcola y Lattuada (2017).
} 
o segmentadas de apoyo a las cooperativas según sean las condiciones de estas. Para las cooperativas de la agricultura familiar, o las diversas formas pre-cooperativas, son necesarias políticas de fomento que simplifiquen los trámites de constitución y algunas documentaciones contables y técnicas que estas deben presentar durante su funcionamiento. Pero esto no es suficiente. Se requieren medidas de asesoramiento técnico y económico para que esos proyectos asociativos sean viables en el tiempo. Y allí es necesario articular de manera coherente con otras agencias estatales que trascienden el sector cooperativo, tales como las agropecuarias e impositivas.

En cambio, para las cooperativas empresariales que se constituyen en importantes unidades del agronegocio, es necesario gestionar otro tipo de políticas públicas. En relación con esto es preciso mejorar los instrumentos normativos para que la legislación cooperativa sea un facilitador para la inserción competitiva en los mercados capitalistas internacionales. Me refiero puntualmente a la necesidad de profundizar la inserción de las cooperativas en las cadenas de agregado de valor y en la exportación directa. No obstante, esto supone transformaciones organizativas e institucionales que pueden tensionar la estructura de las cooperativas y contradecir algunas cuestiones que los socios consideren sustanciales.

\section{Referencias bibliográficas}

1. Bohoslavsky, E. y Soprano, G. (2010). Un Estado con rostro humano. Funcionarios e instituciones estatales en Argentina (desde 1880 a la actualidad). Prometeo.

2. Girbal de Blacha, N. (2002). Políticas públicas para el agro se ofrecen. Llamar al estado peronista. Mundo Agrario, 3 (5), 1-20.

3. Girbal de Blacha, N. (2003). Mitos, paradojas y realidades en la Argentina peronista (1946-1955). Una interpretación histórica de sus decisiones políticoeconómicas. Universidad Nacional de Quilmes.

4. Lattuada, M. (2002). El peronismo y los sectores sociales agrarios. La resignificación del discurso como articulador de los cambios en las relaciones de dominación y la permanencia en las relaciones de producción. Mundo Agrario, 3 (5), 1-20.

5. Lattuada, M. (2006). Acción colectiva. Corporaciones agrarias en la Argentina. Transformaciones institucionales a fines del siglo XX. Universidad Nacional de Quilmes.

6. Lattuada, M., Nogueira, M. E., Renold, J. M., Urcola, M. (2011). Cooperativismo y capital social en la agricultura argentina a comienzos del siglo XXI. Programa de Servicios Agrícolas Provinciales-Ministerio de Agricultura, Ganadería y Pesca.

7. Lattuada, M. $\left(2014^{\mathrm{a}}\right)$. Las asociaciones económicas no cooperativas de la agricultura familiar. Estudios de casos y lecciones aprendidas en sus procesos de 
surgimiento y consolidación. Instituto Nacional de Tecnología Agropecuaria/ Instituto Interamericano de Cooperación para la Agricultura.

8. Lattuada, M. $\left(2014^{\mathrm{b}}\right)$. Las asociaciones económicas no cooperativas de la agricultura familiar. Algunas nociones y conceptos para su estudio $y$ consolidación. Instituto Nacional de Tecnología Agropecuaria/ Instituto Interamericano de Cooperación para la Agricultura.

9. Mateo, G. (2012). Cooperativas agrarias y peronismo. Acuerdos y discrepancias. La Asociación de Cooperativas Argentinas. Ediciones del Centro de Integración, Comunicación, Cultura y Sociedad.

10. Nogueira, M. E., Urcola, M. y Lattuada, M. (2017). La gestión estatal del desarrollo rural y la agricultura familiar en Argentina: estilos de gestión y análisis de coyuntura 2004-2014 y 2015-2017. Revista Latinoamericana de Estudios Rurales, // (4), pp. 23-59.

11. Olivera, G. (2015). Cooperativas y gremios chacareros como piezas claves en la policía agraria peronista. En O. Graciano y G. Olivera (Comp.) Agro y política en la Argentina. Tomo II: Actores sociales, partidos políticos e intervención estatal durante el peronismo 1943-1955. Ediciones del Centro de Integración, Comunicación, Cultura y Sociedad.

12. Pucciarelli, A. y Castellani, A. (2014). Los años del kirchnerismo. La disputa hegemónica tras la crisis del orden neoliberal. Siglo Veintiuno. 